\title{
SUITABILITY FOR DAY CASE CATARACT SURGERY
}

\author{
K. J. LOWE, D. A. GREGORY, R. I. JEFFERY, D. L. EASTY \\ Bristol
}

\begin{abstract}
SUMMARY
Areas of concern about day case surgery are highlighted. A group of $\mathbf{4 4 2}$ patients underwent cataract surgery with lens implant. They were randomly allocated to day case or inpatient groups. Questionnaires were used to assess opinions about day case cataract surgery and how patients felt they would manage. None of the areas of concern were actually a problem. Difficulties encountered by patients and clinicians in the postoperative period are discussed. Most patients appear suitable for day case surgery provided they are well informed.
\end{abstract}

Although day case cataract surgery is performed in many centres in the UK the selection criteria are varied and often restrictive. This denies large numbers of patients the option of day case surgery. In many cases the criteria are purposely overprotective due to lack of experience and available data. An optimistic target of $20 \%$ of all cataract surgery performed as day cases has been suggested by the Audit Commission. ' In view of the variation in selection criteria is this target realistic?

It has been shown that early mobilisation does not alter the outcome of cataract surgery. ${ }^{2}$ Our experience with day case cataract surgery suggests that in fact there are few barriers to treating patients as day cases. Also as the concept of day case surgery is spread into the community many patients would rather be treated this way. ${ }^{3}$

The primary concern about day case cataract surgery is patient safety and prompt treatment of any complications. Cataract surgery itself is not physically disabling. Most patients can rely on their unoperated eye in the immediate postoperative period and this is often their better eye. Also it is not unusual for the operated eye to see more clearly after the first dressing. Hence visually the patient is unlikely to be at a disadvantage. Local anaesthesia is not systemically disabling and patients in some centres are mobilised immediately from the operating table. Supervision in most hospital wards is not as intense as it once was when patients were confined to bed for many days. Previous work looking at postoperative confusion and

From: University Department of Ophthalmology, Bristol Eye Hospital, Bristol, UK.

Correspondence to: Mr K. J. Lowe, Sunderland Eye Infirmary, Queen Alexandra Road, Sunderland SR2 9HP, UK. psychosis following cataract surgery suggests a familiar environment is advantageous. ${ }^{4}$ Also accidents may be less likely in a familiar home environment.

Do patients who are coping with or without support need additional physical help or supervision after a cataract operation? Provided patients and any helpers are well informed, is knowing that they can contact or return to the hospital sufficient?

The main areas of concern when selecting patients for day case surgery are: (1) living alone, (2) lack of supervision, (3) availability of help, (4) eyedrop administration, (5) running the home, (6) access to a telephone, (7) transport and (8) recognition and management of any complications.

\section{MATERIALS AND METHODS}

Patients were recruited from six of the seven cataract waiting lists at the Bristol Eye Hospital. The criteria for selection were as follows:

\section{Inclusion criteria}

1. Living within an 8 mile $(13 \mathrm{~km})$ radius of the hospital.

2. Listed for cataract extraction with lens implant.

3. Aged 55 years or over.

4. Informed consent.

Exclusion criteria

1. Only one potentially good eye.

2. Previous intraocular procedure on the same eye.

3. Ocular pathology complicating cataract extraction.

4. Listed for general anaesthesia.

Patients meeting the above criteria were invited to attend a preoperative assessment clinic. They were randomly allocated to day case or inpatient groups before being seen. Those who agreed to be included in the trial were informed of their group. Apart from the ophthalmic assessment the history and examination were concerned with fitness for local anaesthesia. Attention was paid to the respiratory system, cardiovascular system and spinal curvature. If necessary the patient's ability to remain supine or keep still was assessed. No routine investigations were performed except urinalysis. Patients were given a date for their operation at the assessment clinic. This was usually within 2-6 weeks. 
All operations were performed by the same surgeon using a standard endocapsular technique with posterior chamber intraocular lens implantation. All patients had local anaesthesia using a posterior peribulbar block. No routine premedication was given preoperatively apart from eyedrops. Preoperative sedation can reduce the level of consciousness and we felt it was safer to have patients orientated while on the operating table. Despite this some patients were relaxed enough to fall asleep during their operation; fortunately none woke up abruptly. Also we preferred patients to be alert and orientated before early discharge into a potentially unsupervised environment.

Prior to the operation patients completed a questionnaire presented by the study's medical social worker. A similar questionnaire was also presented 16 weeks after the operation.

Inpatients remained in hospital for 2 days after their operation. The surgeon examined their eyes each morning using a Haag Streit slit lamp and applanation tonometry. After the first postoperative examination there were no restrictions on ambulation. After the second examination patients were discharged.

Day cases were visited in their home on the first two mornings after their operation. The study's ophthalmic nurse examined theireyes using a portable slit lamp and a Tono-pen. The Tono-pen is a portable device for measuring intraocular pressure based on the strain gauge principle. It compares well with Goldmann applanation tonometry over the normal range of intraocular pressure and up to $30 \mathrm{mmHg}$. ${ }^{5,6}$

Day cases completed a questionnaire on the second postoperative day presented by the study's nurse. This outlined how they felt they had managed and what degree of support they had on returning home.

\section{RESULTS}

Of the 448 patients who attended the assessment clinic 442 were given a date for surgery. Six were excluded and taken off the cataract waiting list. One had a retinal detachment behind the cataract, 1 had a macular hole with minimal opacity, 1 had a disciform macular degeneration with minimal opacity and 3 did not have significant opacities. Two hundred patients were managed as day cases and 242 as inpatients.

A compromise was necessary in the positioning of a few patients on the operating table due to breathing difficulties

Table I. Intraocular pressure on the first postoperative day

\begin{tabular}{lcc}
\hline $\begin{array}{l}\text { Intraocular pressure } \\
(\mathrm{mmHg})\end{array}$ & Day cases & Inpatients \\
\hline$<22$ & 185 & 233 \\
$22-25$ & 7 & 6 \\
$26-30$ & 4 & 3 \\
$31-35$ & 0 & 0 \\
$36-40$ & $1^{\text {a }}$ & 0 \\
$40-45$ & 1 & 0 \\
Total & 198 & 242 \\
\hline
\end{tabular}

In 2 cases we were unable to check the intraocular pressure.

${ }^{\text {a }}$ Patient with primary open angle glaucoma. or spinal curvature. Hiatus hernias and arthritis also caused minor problems for some patients when supine. No patients were excluded or had their operation cancelled because of their general health.

No operations were cancelled due to local anaesthetic complications. There were no retrobulbar haemorrhages and no additional injections were required. Lid akinesia was complete in $87 \%$, minimal twitching was observed in $9 \%$ and significant movement in $4 \%$. Eye movement was absent in $76 \%$, minimal in $19 \%$ and significant in $5 \%$. Patients assessed any preoperative pain on a weighted analogue scale: $88 \%$ felt no pain, $10 \%$ felt mild pain, $2 \%$ felt moderate pain and none felt severe pain.

One day case was kept in overnight as a precaution after a threatened expulsive haemorrhage. Two serious problems occurred after discharge in the day case group. These were an infective endophthalmitis and an iris prolapse, both diagnosed by the ophthalmic nurse on the postoperative visits. The endophthalmitis responded poorly to treatment and was ultimately enucleated. The iris prolapse was repaired without further problems.

In the inpatient group there were two early postoperative problems: an inflammatory endophthalmitis which responded to steroid treatment and an iris prolapse which was successfully repaired.

Intraocular pressure was raised on the first postoperative day in 13 day cases and 9 inpatients (Table I). No patients had a raised intraocular pressure on the second day or any long-term problems.

The number of patients attending casualty over the 16 weeks following their operation was similar in day cases $(9.5 \%)$ and inpatients $(8.7 \%)$. However, inpatients attended more frequently (37 times for 21 inpatients against 21 times for 19 day cases; Table II). No day case patients attended casualty during the first two postoperative days. Eight patients who attended casualty had no obvious abnormality and were reassured. Two patients had unrelated problems with their other eye; one inpatient with a fibrin membrane was admitted from casualty on the fifth postoperative day. No serious problems presented to casualty.

Preoperative questionnaires were completed by 431 patients (193 day cases and 238 inpatients). The day cases had an average age of 74.2 years (SD 8.7) and a male to

Table II. Reasons for casualty attendance within 16 weeks of operation

\begin{tabular}{lcc}
\hline & Day cases & Inpatients \\
\hline Allergic reaction & 1 & 3 \\
Fibrin membrane & 0 & $3^{\mathrm{a}}$ \\
Raised IOP (steroids) & 0 & 1 \\
No abnormality & 4 & 4 \\
Pain & 2 & 1 \\
Subconjunctival haemorrhage & 2 & 1 \\
Suture/irritation & 1 & 1 \\
Uveitis & 4 & 5 \\
Visual acuity & 2 & 2 \\
Watery eye & 3 & 0 \\
Total & 19 & 21 \\
\hline
\end{tabular}

${ }^{\text {a }}$ One inpatient was admitted via casualty. 
Table III. Patients` primary complaint about health

\begin{tabular}{lcr}
\hline & Day cases & Inpatients \\
\hline Arthritis & $23(51)^{4}$ & $36(42)$ \\
Cardiovascular problems & $3(1)(80)$ & $24(86)$ \\
Diabetes & $12(33)$ & $8(30)$ \\
Respiratory problems & $13(18)$ & $16(16)$ \\
\hline
\end{tabular}

"Figures in brackets refer to significant history of disease and/or current treatment for the condition.

female ratio of $77: 116$. The inpatients had an average age of 75.2 years (SD 7.9) and a male to female ratio of $90: 148$. The following information relates to all patients except where significant differences exist regarding age, sex, living alone or treatment groups.

Questionnaires were used to assess the patients' view of their general health and their primary health problem (Table III).

Patients were asked about caring for themselves and their eye after the operation. For self care $94.4 \%$ were confident and $5.6 \%$ were not. For eye care $92.3 \%$ were confident and $7.7 \%$ were not. For drop instillation $83.3 \%$ felt they would probably or definitely be able to cope, while $16.7 \%$ felt they would need help.

One hundred and eighty patients lived alone, of whom $50.6 \%$ received help with one or more of the activities of daily living. Two hundred and fifty-one patients lived with another adult; of these $52.2 \%$ received help (Table IV). More women lived alone than men $(52.3 \%$ and $24.6 \%$ respectively). The number of women living alone steadily increased with age over 65 and more steeply after 85 . The number of men living alone increased slightly over the age of 80 .

All males and $94.8 \%$ of females who lived with another adult had a potential helper postoperatively. Of those who lived alone $48.8 \%$ of males and $74.5 \%$ of females had a potential helper. Only $14.2 \%$ of patients thought they would have to manage without help. A breakdown by treatment is given (Table V). One hundred and forty day cases had help during the first 2 days after surgery (Table VI). Only 76 actually wanted help. Fifty-three day cases managed alone, 5 of whom would have liked help.

Analysis of our day cases showed that $91.5 \%$ could have been treated as either a day case or an inpatient without any obvious advantage. Only 5\% would have benefited by being a day case and $3.5 \%$ by being an inpatient. Of the 193 day cases $87 \%$ were happy to be in this group

Table IV. Patients receiving help with the activities of daily living

\begin{tabular}{lcc}
\hline & L.ive alone & Not alone \\
\hline Cooking & 17 & 54 \\
Cleaning & 5() & 68 \\
Laundry & 48 & 57 \\
Shopping & 66 & 80 \\
Personal care & 11 & 24 \\
Emotional support & 27 & 46 \\
Pension & 39 & 39 \\
Prescription & 33 & 41 \\
Accompanied outside & 29 & 49 \\
\hline
\end{tabular}

Many patients received help with more than one activity.
Table V. Numbers of patients who thought a helper would be available after the operation

\begin{tabular}{lll}
\hline & \multicolumn{2}{c}{ Help available } \\
\cline { 2 - 3 } & Yes & No \\
\hline Day cases & 168 & 25 \\
Inpatients & 200 & 36 \\
Total & 368 & 61 \\
\hline
\end{tabular}

preoperatively, improving to $94 \%$ postoperatively $(p<0.05)$. For the 238 inpatients the figures were $83 \%$ and $88 \%$ respectively; the change was not significant.

All patients felt the care given before and after operation was adequate or good. Two per cent of patients did not feel the explanation of the operation was adequate.

\section{DISCUSSION}

Many of our patients lived alone. Help was available for most of them; however some preferred to cope alone. Only a small number who felt they required help did not have any, and they managed nevertheless. We tend to underestimate both the degree of independence and the availability of short-term support for the elderly. Nearly half the day cases who had help did not feel they needed it. This is not surprising since a cataract operation is not physically disabling.

Patient and helper education was an important factor in encouraging independence and allaying anxiety. A much simplified drop regime was also useful. We prescribed Betnesol-N drops (Glaxo, Greenford, Middx.) t.i.d. for virtually all our patients, reasoning that compliance would be more likely with a simple regime. Complicated multiple-drop regimes probably cause more anxiety than anything else in the postoperative period.

Many of our patients did not have telephones; usually a neighbour or friend did and could be relied upon. We did not, however, specify this as a requirement of a patient having day case surgery.

Only 2 of our patients did not arrange their own transport as requested. One insisted on walking home and the other caught a bus. The remainder arranged a lift or a taxi. It was common for patients from the same area to help one another with transport. Several lasting friendships were formed in this manner. This was an unexpected benefit of grouping patients to make postoperative home visits more efficient. The hospital car service could have been utilised for clinic attendance, but was not available at the required time for the day of operation.

Many patients commented that they were happier in

Table VI. Persons who provided help for 14() day cases on the tirst two postoperative days

\begin{tabular}{lr}
\hline Helper & \multicolumn{1}{c}{$\%$} \\
\hline Spouse & 56 \\
Son/daughter & 21 \\
Brother/sister & 6 \\
Other relative & 4 \\
Friend/neighbour & 13 \\
\hline
\end{tabular}


their home environment than in hospital. However, not everyone follows the recommendations given when discharged. Does keeping them in hospital for an extra day or two make a difference? In our study 2 patients traumatised their eye. One inpatient poked himself in the eye before discharge from hospital, breaking a suture. Three weeks after discharge another patient bent down and hit his eye on the corner of a table, rupturing the wound and expelling the intraocular lens.

It is clear that help is available from friends and relatives in many cases. The few people who felt they needed help were generally referring to drop instillation. In reality feeling you need or would like help and not being able to manage are not necessarily the same. It could also be argued that not all those who think they can manage will.

One of our main concerns was postponing operations due to anaesthetic problems, in particular retrobulbar haemorrhage. This complication is thought to be unlikely with posterior peribulbar anaesthesia and our experience supports this view.?

Complications are fortunately rare in cataract extraction. Most occur during the operation or as a consequence of poor technique. Many problems can occur after the first postoperative day. When is it totally safe to discharge a patient? The risks seem acceptably small for immediate discharge. ${ }^{2}$ The only possible exception is an infective endophthalmitis, a rare but potentially devastating complication. In theory it could be picked up in an inpatient prior to the planned first dressing, but in reality is it? Two of our patients had an intraocular pressure above $30 \mathrm{mmHg}$. Both were day cases, one of whom had primary open angle glaucoma. Both cases were picked up with the Tono-pen; one had mild corneal oedema, the other appeared normal on slit lamp examination.

The second postoperative home visit did not disclose any additional problems. Hence we do not feel a routine second visit necessary in the majority of day cases. The option of a second visit is useful and would have been employed in the two patients with intraocular pressures above $30 \mathrm{mmHg}$.

Many day case patients in other centres are asked to return for an examination the following morning. There is a risk that a patient may not return after the operation. This did not occur with any of our patients at the 2-week visit. However, several patients with good initial vision defaulted from further visits. We think that the incentive to be seen on day 1 to be reassured that everything is satisfactory is so strong that few if any patients would be likely to default. It is an advantage to be able to send out someone to examine the eye in this respect.

The majority of patients could have been managed as either a day case or an inpatient without any obvious advantage or disadvantage to them. A minority would have been more suited to one method of management due to strong preference or home conditions. In practice any benefit was marginal.

Time and trouble invested in educating patients about treatment and postoperative care cannot be overemphasised. It is here that our nurse practitioner was probably most effective. Written literature is also useful; however, some answers to our questionnaires suggested it was misunderstood or had not been read. It is useful if any relative or friend who will be helping in the postoperative period can attend the assessment clinic with the patient. In some cases helpers seem less inhibited than the patient, voicing areas of concern. They can also help the patient to understand and follow any recommendations.

Few patients feel strongly about being a day case or an inpatient. Outside the study it is the authors' experience that patients who are offered a choice of day case or inpatient management often ask which their doctor would recommend. Others choose inpatient surgery because of the option of general anaesthesia. The majority would not gain any significant benefit by being treated as a day case or an inpatient. In view of this we would recommend a relaxation in the stringent criteria initially employed once a day case service has been established. Naturally not all patients will be treated as day cases and some day cases may require inpatient admission. However, for those who wish to do day case surgery the majority of the patients on the waiting lists are suitable. The results of day case surgery and the satisfaction of the patients will soon create a demand for this type of treatment within the local community.

This work was funded by the Medical Research Council grant no. G8621843.

Key words: Day case, Independence, Inpatient, Patient selection, Support.

\section{REFERENCES}

1. Audit Commission: A short cut to better services. Day case surgery in England and Wales. First report. London: HMSO, 1990.

2. Galin MA, Baras I, Barasch K, Boniuk V: Immediate ambulation and discharge after cataract surgery. Trans Am Acad Ophthalmol Otol 1974, 78: 43-9.

3. Lowe KJ, Gregory DA, Jeffery RI, Easty DL: Patient perceptions and social impact: preliminary report of the Bristol MRC study. Eye 1991, 5: 373-8.

4. Lin L: Psychiatric reactions complicating cataract surgery. Int Ophthalmol Clin 1965, 5: 143-54.

5. Minckler DS, Baerveldt G, Heurer DK, et al. Clinical evaluation of the Oculab Tono-pen. Am J Ophthalmol 1987, 104: 168-73.

6. Frenkel RE, Hong YJ, Shin DH: Comparison of the Tono-pen to the Goldmann applanation tonometer. Arch Ophthalmol 1988, 106: 750-3.

7. Davis DB, Mandel MR: Posterior peribulbar anaesthesia: an alternative to retrobulbar anaesthesia. $J$ Cataract Refract Surg 1986, 12: 182-4. 